\title{
Influence of squeeze-film damping on higher-mode microcantilever vibrations in liquid
}

\author{
Benjamin A Bircher ${ }^{*}$, Roger Krenger and Thomas Braun ${ }^{*}$
}

\author{
* Correspondence: \\ benjamin.bircher@unibas.ch; \\ thomas.braun@unibas.ch \\ Center for Cellular Imaging and \\ NanoAnalytics, Biozentrum, \\ University of Basel, Mattenstrasse 26, \\ $\mathrm{CH}-4058$ Basel, Switzerland
}

\begin{abstract}
Background: The functionality of atomic force microscopy (AFM) and nanomechanical sensing can be enhanced using higher-mode microcantilever vibrations. Both methods require a resonating microcantilever to be placed close to a surface, either a sample or the boundary of a microfluidic channel. Below a certain cantilever-surface separation, the confined fluid induces squeeze-film damping. Since damping changes the dynamic properties of the cantilever and decreases its sensitivity, it should be considered and minimized. Although squeeze-film damping in gases is comprehensively described, little experimental data is available in liquids, especially for higher-mode vibrations.

Methods: We have measured the flexural higher-mode response of photothermally driven microcantilevers vibrating in water, close to a parallel surface with gaps ranging from $\sim 200 \mu \mathrm{m}$ to $\sim 1 \mu \mathrm{m}$. A modified model based on harmonic oscillator theory was used to determine the modal eigenfrequencies and quality factors, which can be converted into co-moving fluid mass and dissipation coefficients.

Results: The range of squeeze-film damping between the cantilever and surface decreased for eigenfrequencies (inertial forces) and increased for quality factors (dissipative forces) with higher mode number.

Conclusions: The results can be employed to improve the quantitative analysis of AFM measurements, design miniaturized sensor fluid cells, or benchmark theoretical models.

Keywords: Microcantilever; Dissipation; Squeeze-film damping; Higher eigenmode; Photothermal excitation; Eigenfrequency; Quality factor; Fluid-structure interaction

PACS: 07.10.Cm (Micromechanical devices and systems); 46.40.Ff (Resonance and damping of mechanical waves); 07.79.-v (Scanning probe microscopes and components); 07.07.Df (Sensors (chemical; optical; electrical; movement; gas; etc.); remote sensing)
\end{abstract}

\section{Introduction}

Damping is an important design criterion for micro- and nanometer sized resonators, because surface forces dominate body forces at small dimensions [1]. Immersing a resonator, e.g., a microcantilever, in fluid drastically changes its dynamic properties. The eigenfrequencies and quality factors decrease due to hydrodynamic forces, which can be decomposed into an inertial (added mass) and dissipative (viscous damping) term [2]. Additionally, placing the resonator close to a solid surface leads to squeezefilm damping, where displacement of the fluid between the resonator and the surface during each vibration period introduces additional added mass and viscous damping [3]. The damping occurring by both mechanisms has direct impact on atomic force 
microscopy (AFM) and dynamically operated nanomechanical sensors. With progressing miniaturization, squeeze-film damping starts to dominate other dissipative effects and, thus, needs to be considered and characterized [4].

Furthermore, higher modes of vibration are increasingly used. In multifrequency AFM imaging, higher modes allow the material characteristics, e.g., mechanical, magnetic or electrical properties, of the substrate to be measured [5]. To reduce squeeze-film damping, AFM samples have been placed on pillars [6], or cantilever geometries have been optimized by focused-ion beam milling [7]. In cantilever-based sensor applications, the use of higher vibrational modes provides increased mass sensitivity [8] and allows the elastic properties [9] and the position of adsorbates [10] to be disentangled. Squeeze-film damping needs to be considered below a certain critical dimension of the AFM cantilever tip or container in which the cantilever sensor is mounted.

To our knowledge, squeeze-film damping of micrometer-sized cantilevers vibrating in higher modes in liquid has not been measured to date. In contrast, it has been thoroughly investigated for resonators immersed in gases, because of its importance for micro-electromechanical systems (MEMS), e.g., torsional mirrors [11] or cantilevers [12]. Even though, less attention has been paid to the problem in liquids, both analytical and numerical methods have been employed to model the behavior of cantilevers immersed in liquid and vibrating in close proximity to a surface. Analytical approaches [13] account for dissipative and inertial effects in the liquid, but due to the assumption of a two-dimensional flow field higher modes of vibration were not considered. Numerical approaches can effectively describe different cantilever-surface inclination angles, vibrational modes, and varying external driving forces [2,4,14]. However, semi-analytical equations describing the hydrodynamic load acting on cantilevers under squeeze-film damping only consider the fundamental mode of vibration [13,14]. Squeeze-film damping in liquid is governed by two dimensionless quantities, the Reynolds number, $R e$, and the normalized gap, $H$ [13]:

$$
R e=\frac{\pi \rho_{f} f_{n} b^{2}}{2 \eta_{f}}, H=\frac{g}{b}
$$

where $b$ is the width of the cantilever, $\rho_{f}$ the fluid density, $\eta_{f}$ the fluid viscosity, $f_{n}$ the cantilever eigenfrequency in liquid, and $g$ the gap between the cantilever and the surface (see Figure 1). The cantilever width, $b$, is the dominant length scale of the flow

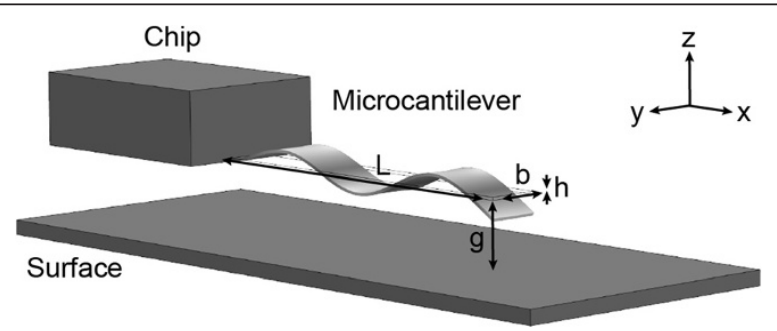

Figure 1 Diagram of a microcantilever vibrating close to a surface. A cantilever of length $L$, width $b$ and thickness $h$, is vibrating at flexural mode $n=4$. The cantilever-surface gap $g$ was varied from $\sim 200 \mu \mathrm{m}$ to $\sim 1 \mu \mathrm{m}$. Liquid confined in the gap causes additional hydrodynamic forces due to squeeze-film damping. The amplitude of vibration is drawn not to scale. 
[13]. If the vibration amplitudes are orders of magnitude smaller than $b$, i.e., KeuleganCarpenter numbers « 1 [15], the effect becomes independent of the amplitude [16]. Furthermore, the continuum hypothesis is valid because the mean-free-path of the molecules in liquid is very small compared to the dominant length $b$ and the gap size $g$, i.e., Knudsen numbers « 1 [15].

Experimental investigations of cantilevers with dimensions ranging from centimeters to micrometers, immersed in water, buffer, organic solvents and oils are reported in the literature [3,15-19]. However, all experimental studies on microcantilevers in liquid and close to a surface, were limited to the fundamental mode $(n=1)$ [17-19]. Here we present the full spectral response of microcantilevers vibrating in water at different distances from a polydimethylsiloxane (PDMS) surface. PDMS was selected because of its abundant use for the fabrication of microfluidic devices. Spurious-free resonance spectra were obtained by driving the microcantilevers photothermally [20], and several higher flexural modes of vibration were characterized.

\section{Results and discussion}

To measure the effects of squeeze-film damping, a tipless microcantilever $(250 \mu \mathrm{m} \times$ $35 \mu \mathrm{m} \times 2 \mu \mathrm{m}$ ) was placed close to a surface and the gap, $g$, was varied from $\sim 200 \mu \mathrm{m}$ to $\sim 1 \mu \mathrm{m}$ using a motorized stage (Figure 1). Experiments using longer cantilevers are reported in the Additional file 1. Photothermal excitation was employed to drive the microcantilever to resonance. Amplitude and phase spectra were acquired by sweeping an excitation frequency range from $0.5 \mathrm{kHz}$ to $800 \mathrm{kHz}$ and recording the corresponding cantilever response. As shown in Figure 2 for different cantilever-PDMS surface gaps, the spectra span four flexural modes of vibration. The influence of the cantileversurface gap became substantial for $H=g / b<1$, causing the resonance peaks to shift towards lower frequencies and broaden significantly (decreasing quality factors). A model, consisting of a sum of damped harmonic oscillators and terms considering the measurement setup (see Methods, Equation 4), described the experimental phase data with good accuracy (Figure 3). Applying this model to the data, allowed the eigenfrequency, $f_{n}$, and quality factor, $Q_{n}$, of each mode $n$, to be extracted at different cantilever-surface gaps, $g$ (Figure 4).

To compare cantilevers from different chips, $f_{n}$ and $Q_{n}$ were normalized to the values indicated by experimental data recorded far from the surface, where its presence had no influence (see Methods). As shown in Figure 5a, due to squeeze-film damping the eigenfrequencies of all modes decrease as the cantilever-surface gaps become smaller. Further, even though some of the differences are slight, it is clear that higher-mode eigenfrequencies are less influenced by the proximity of the surface. In contrast, the higher-mode quality factors are affected when the cantilever-surface gap is still comparatively large (Figure $5 \mathrm{~b}$ ) and the fundamental mode is influenced least. To quantitatively compare the effects, a characteristic cantilever-surface gap $g_{n}{ }^{*}$ was defined for the fundamental mode as $g_{1}{ }^{*}=b / 2=17.5 \mu \mathrm{m}\left(H_{1}{ }^{*}=0.5\right)$. At $g_{1}{ }^{*}$ the frequencies (mean $\pm \mathrm{SD}$ ) of the fundamental vibration dropped to $(93.4 \pm 0.7) \%$ and the quality factors to $(77.8 \pm 7.5) \%$ of the initial value. Corresponding characteristic gaps $\left(g_{n}^{*}\right)$, where the frequencies and quality factors dropped by the amounts measured for $g_{1}$ *, were then determined for the higher modes of vibration. As shown in Figure 6, the 


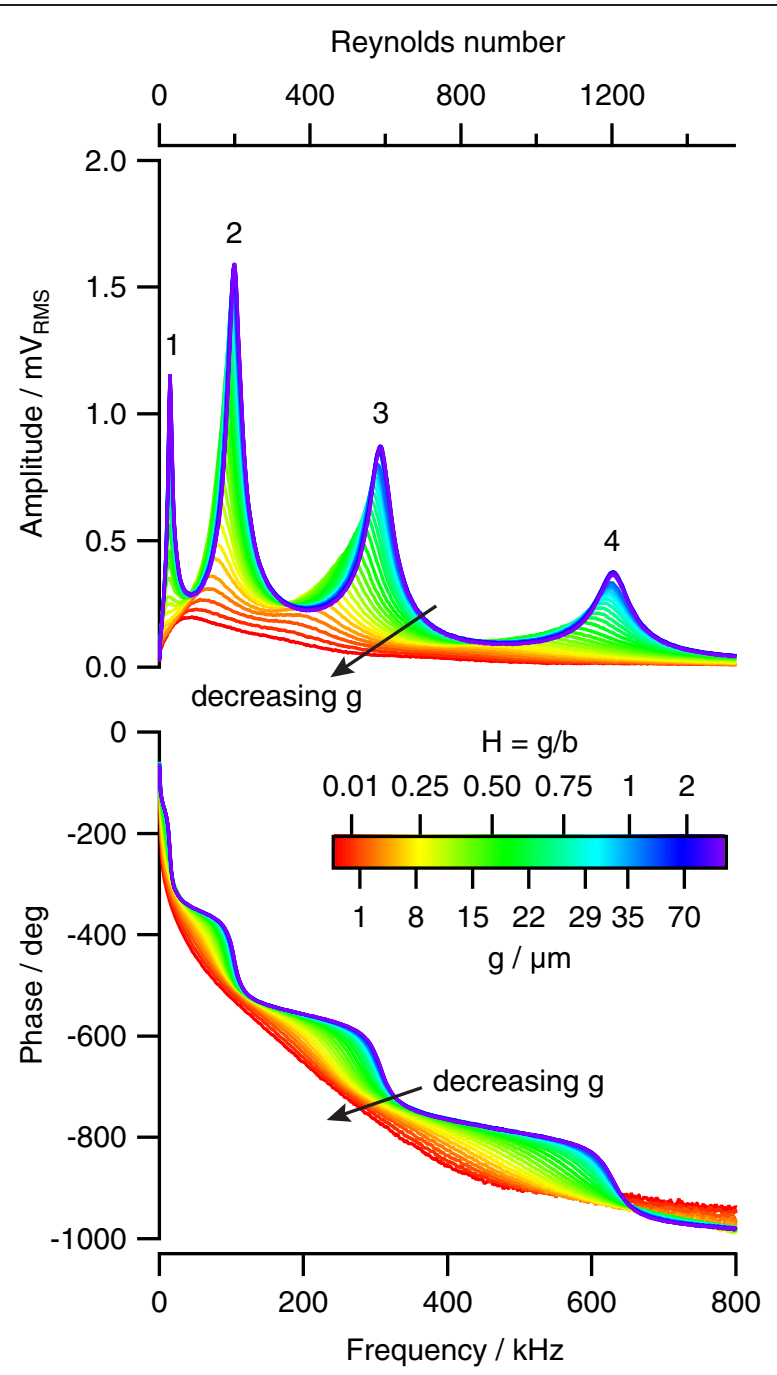

Figure 2 Amplitude and phase spectra of a microcantilever vibrating at different gaps $g$ to a surface. The amplitude (upper plot) and phase (lower plot) response of a microcantilever ( $250 \mu \mathrm{m} \times 35 \mu \mathrm{m} \times$ $2 \mu \mathrm{m}$ ) vibrating in water at different distances from a surface are shown as a function of frequency and the corresponding Reynolds number (Re). The flexural mode numbers are written above the resonance peaks. Absolute $(g)$ and normalized $(H)$ cantilever-surface separations are indicated. The color scale is not linear; far from the surface the increment in $g$ was set larger because the effect diminishes (superimposed purple curves). The spectra are overlayed; the red curves (small $g$ ) are at the back.

characteristic gap decreases for the eigenfrequencies and increases for the quality factors with increasing mode number. Similar behavior was observed for longer cantilevers (see Additional file 1), however, the effect seems to diminish with increasing cantilever length. To estimate the range of squeeze-film damping, i.e., the gap where the onset of the effect occurs, the characteristic gap was multiplied by a factor of two. Because of the definition, the range of squeeze-film damping for the fundamental mode is $H=1$. The ranges for modes 2 to 4 ( mean $\pm \mathrm{SD}$ ) were $0.93 \pm 0.18,0.84 \pm 0.21$, and $0.74 \pm 0.21$ for the eigenfrequencies and $1.22 \pm 0.35,1.55 \pm 0.37$, and $1.67 \pm 0.20$ for the quality factors. The largest critical gap for the frequencies, i.e., where the surface has no influence on the dynamics of the microcantilever, can be estimated from the fundamental mode, 


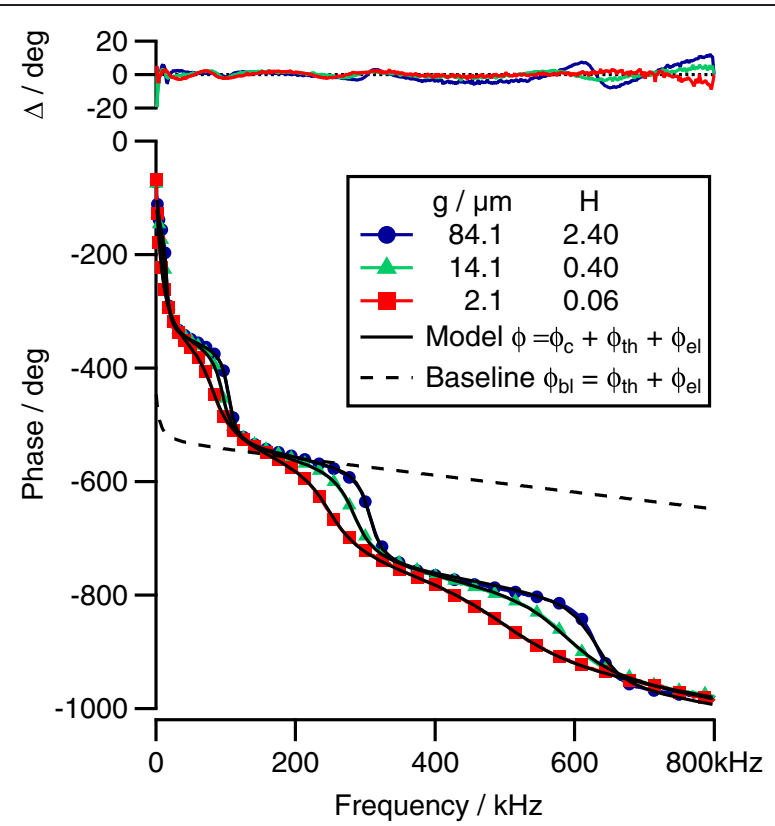

Figure 3 Phase model and data. Lower plot: Phase data recorded at three different cantilever-surface gap heights (only every twentieth marker is shown), the model $\phi$ (solid black line) and the baseline $\phi_{b l}$ (dashed black line) included in the model to account for the phase response of the photothermal excitation $\phi_{t h}$ and the measurement electronics $\phi_{e l}$ Upper plot: The difference between the model and the experimental data, $\Delta$; to extract the eigenfrequencies and quality factors from the data, $\Delta$ was minimized using a Levenberg-Marquardt algorithm.

which is affected first. In contrast, the largest critical gap for the quality factors depends on the highest mode measured.

To obtain a more general description of the results, the added mass coefficient, $a_{m}$, and the damping coefficient, $c$, were calculated for each mode. While the added mass coefficient $a_{m}$ quantifies the co-moving fluid mass relative to the cantilever mass and is

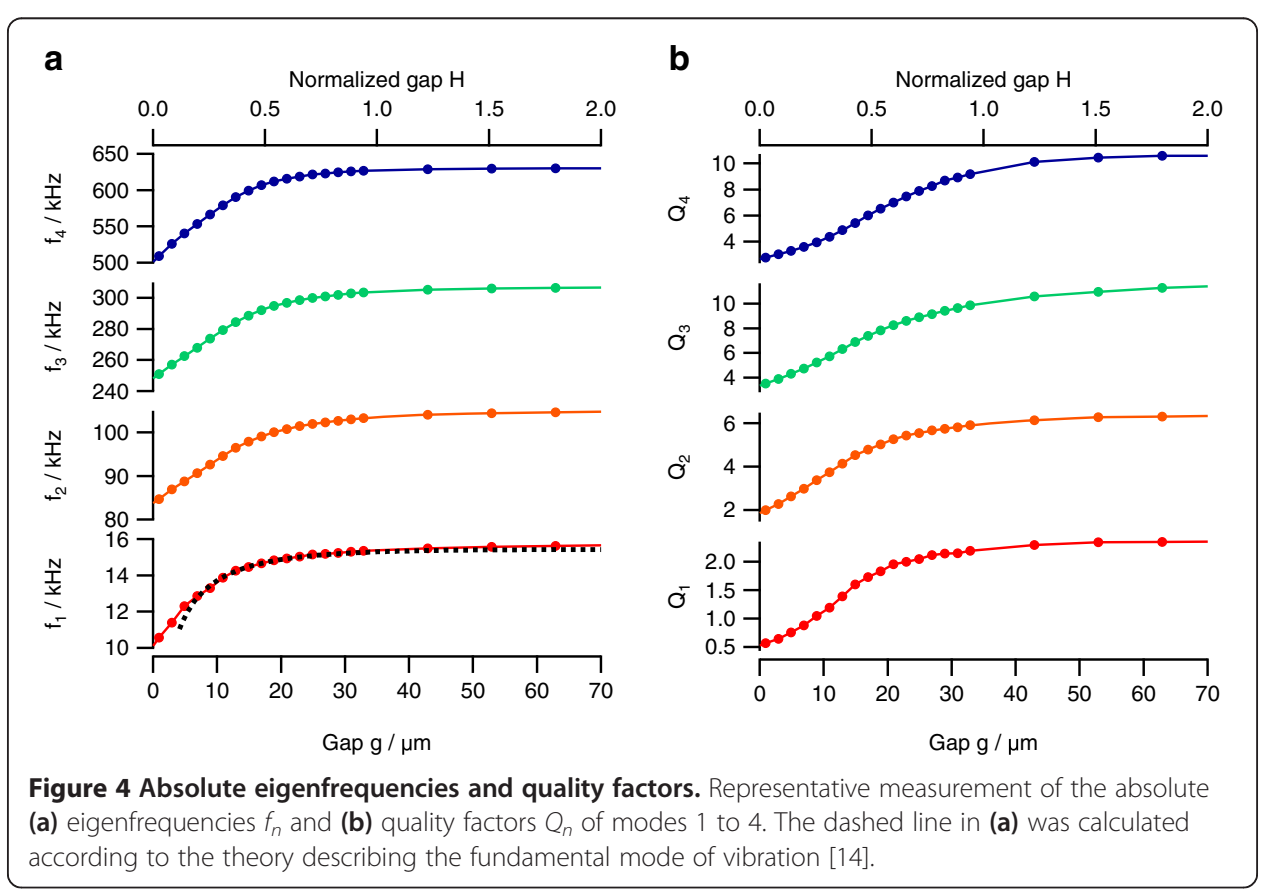



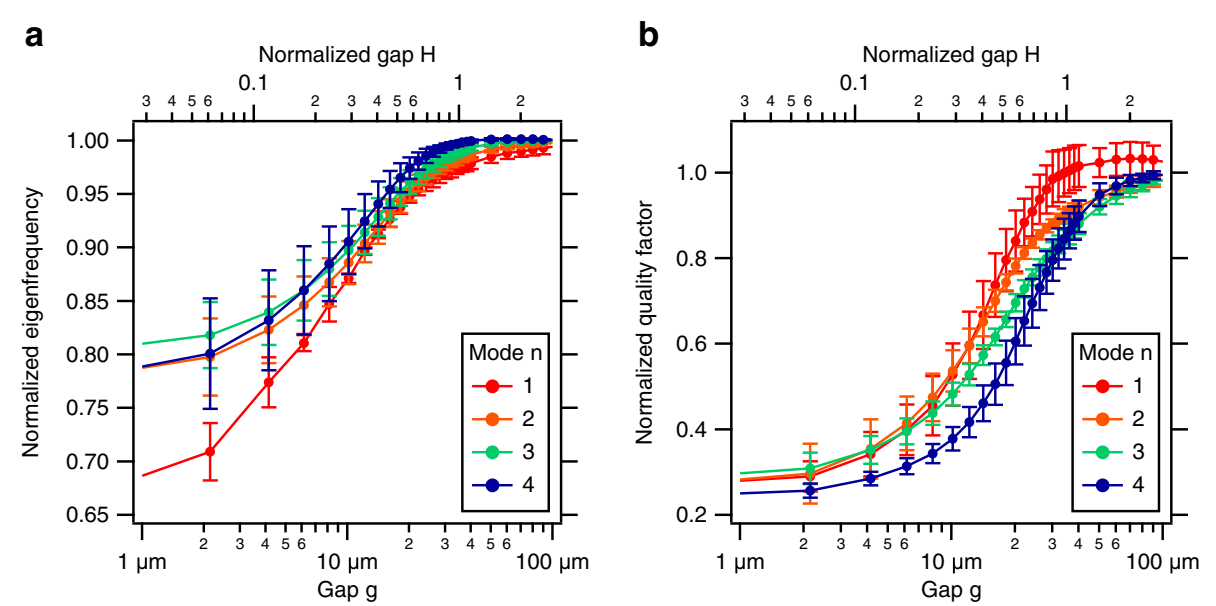

Figure $\mathbf{5}$ Normalized eigenfrequencies and quality factors. (a) Eigenfrequencies and (b) quality factors of a microcantilever, vibrating at different gaps $g$ to a surface. All values were normalized to the values measured far from the surface. The means \pm SD are shown $(\mathrm{N}=3)$.

a measure of the inertial loading, the damping coefficient $c$ equals the energy dissipation per unit length acting on the cantilever. The required vacuum frequencies were calculated using Equations 6 and 7 (see Methods) and the eigenfrequencies recorded in the unbounded fluid, i.e., far from the surface (Table 1). The observed vacuum frequency variations mainly originate from manufacturing-related uncertainties in the

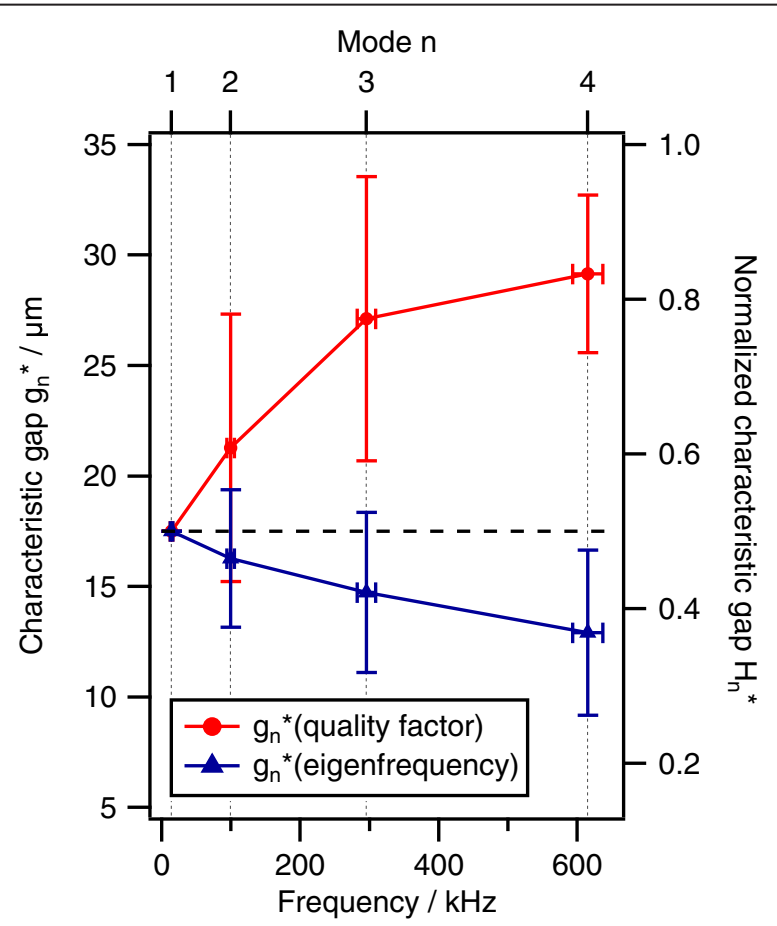

Figure 6 Characteristic gap to estimate the range of squeeze-film damping. The characteristic gap $g_{n}{ }^{*}$ is normalized to the fundamental mode of vibration $(n=1)$ at $H_{1}{ }^{*}=0.5$ (dashed line). It is the cantilever-surface gap, where the frequencies (blue triangles) and quality factors (red circles) dropped to, respectively, $93.4 \%$ and $77.8 \%$ of their initial values. The range of the squeeze-film damping can be estimated by calculating $2 \cdot H_{n}{ }^{*}$, i.e., $H=1$ for the fundamental mode. The means $\pm S D(N=3)$ are shown, mode numbers $n$ are indicated on the top axis. 
Table 1 Vacuum frequencies, added mass coefficients and damping coefficients measured far from the surface (mean \pm SD)

\begin{tabular}{llll}
\hline Mode $\mathbf{n}$ & $\boldsymbol{f}_{\boldsymbol{n}, \boldsymbol{v a c}} / \mathbf{k H z}$ & $\boldsymbol{a}_{\boldsymbol{m}, \boldsymbol{H} \boldsymbol{\prime} \mathbf{1}}$ & $\boldsymbol{c}_{\boldsymbol{H} \boldsymbol{\prime} \mathbf{1}} / \mathbf{m P a} \cdot \mathbf{s}$ \\
\hline 1 & $44.8 \pm 5.4$ & $9.17 \pm 0.20$ & $67.8 \pm 1.6$ \\
\hline 2 & $277 \pm 15$ & $6.78 \pm 0.03$ & $116.2 \pm 11.1$ \\
\hline 3 & $768 \pm 34$ & $5.75 \pm 0.01$ & $186.7 \pm 4.43$ \\
\hline 4 & $1512 \pm 52$ & $5.05 \pm 0.01$ & $372.0 \pm 26.9$ \\
\hline
\end{tabular}

dimensions of the microcantilevers. Subsequently, the added mass coefficients could be determined (see Methods, Equation 6). The values without the influence of squeezefilm damping $(H » 1)$ are provided in Table 1 . Note that some authors defined the added mass coefficient as the co-moving mass relative to the fluid mass displaced by the static cantilever $[3,16]$. For direct comparison with their values, $a_{m}$ has to be multiplied by $\rho_{c} / \rho_{f}$, i.e., $\sim 2.3$ in the present case. The damping coefficients $c$ are the sum of structural, $c_{s}$, and viscous, $c_{v}$ damping. For microcantilevers immersed in liquid, structural damping is orders of magnitude smaller than viscous damping $\left(c_{s} « c_{v}\right)$, and can thus be neglected [3]. The damping coefficients were calculated using the measured quality factors and eigenfrequencies (Equation 8, see Methods). Table 1 shows the damping coefficients without the influence of squeeze-film damping $(H » 1)$. Even though higher-modes dissipate less energy per oscillation cycle (higher quality factors), they have larger damping coefficients due to their higher eigenfrequencies (cycles/second). Figure 7 shows how the added mass and damping coefficients increase due to squeeze-film damping. The magnitude of the observed shift in added mass coefficients decreased with mode number, whereas the shift in damping coefficients increased.
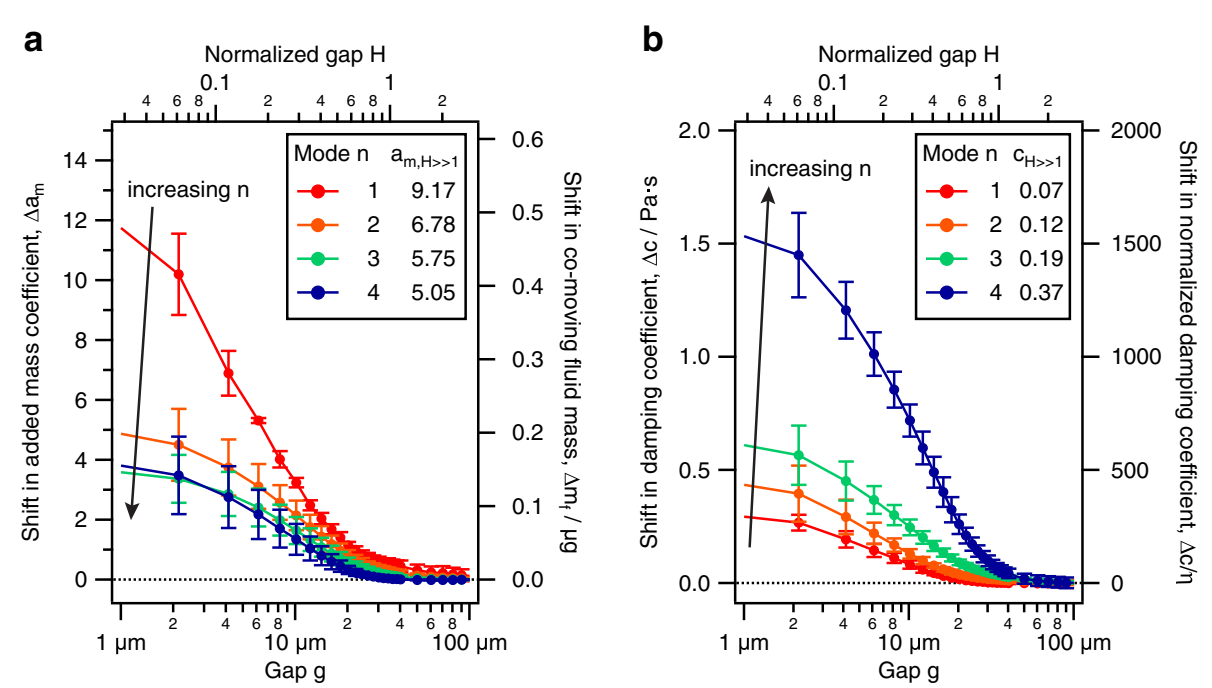

Figure 7 Shift in added mass and damping coefficients due to squeeze-film damping. (a) The shift in the added mass coefficient $\Delta \mathrm{a}_{m}$ quantifies the co-moving fluid mass relative to the cantilever mass and is a measure for the inertial loading; the shift in the absolute fluid mass $\Delta \mathrm{m}_{f}$ is provided on the right axis. (b) The shift in damping coefficient $\Delta c$ equals the dissipation per unit length acting on the cantilever; the shift in dissipation normalized to the fluid viscosity $\eta$ is provided on the right axis. The shifts $\Delta \mathrm{a}_{m}$ and $\Delta \mathrm{c}$ caused exclusively by squeeze-film damping were calculated by subtracting the values recorded in the unbounded fluid, $a_{\left.m, H_{x}\right)}$ and $c_{H \gg 1}$, provided in the figure legend. The means $\pm \mathrm{SD}(\mathrm{N}=3)$ are shown. 


\section{Conclusions}

We have measured the squeeze-film damping on higher flexural mode vibrations of microcantilevers placed in proximity to a parallel surface in liquid. Due to the strong damping only a direct excitation method, such as the employed photothermal excitation [20], obtains spurious-free resonance spectra. A model consisting of a sum of harmonic oscillators was employed to extract the modal eigenfrequencies and quality factors from the phase spectra, and described the measured data well. Correct alignment of the data, i.e., calibration of the gap $g$, was crucial and limited the precision of the measurements. As predicted [13,14], strong squeeze-film damping of the fundamental mode was observed for normalized gaps $H<1$. With increasing mode number the range of squeeze-film damping decreased for the eigenfrequencies (inertial forces) and increased for the quality factors (dissipative forces). Furthermore, the effect seems to depend on the length of the cantilever that determines the spatial wavelength of each mode. These findings should be considered for the design of sensor containers and cantilever tip geometries, because the quality factor is directly related to the sensitivity of the sensor [5]. The observed behavior is likely due to the three-dimensional nature of the flow field generated by higher modes, where gradients along the length of the cantilever must not be neglected [14]. For theoretical models, this entails the introduction of another parameter, besides the normalized gap $H$ and the Reynolds number $R e$, related to the spatial wavelength of the cantilever, i.e., depending on the mode number as well as the cantilever length (similar to the normalized mode number in [21]). Finally, added mass and damping coefficients were calculated to support the comparability of the data. The shift in added mass decreased with mode number as predicted by numerical models [2]. The opposite was observed for the damping coefficients, which increased. More work is required to identify the underlying mechanisms governing squeeze-film damping acting on higher modes. Nevertheless, our data from microcantilevers with common dimensions, allows the magnitude of the squeeze-film damping effect to be assessed.

\section{Methods}

\section{Experimental setup}

Measurements were made as the upper surface of a small cavity containing water was moved closer to the immersed microcantilever. A diagram of the experimental setup is shown in Figure 8. Cantilever vibration was driven by photothermal excitation induced by an intensity-modulated laser beam $(405 \mathrm{~nm})$, and detected by monitoring the deflection of a second laser beam $(780 \mathrm{~nm})$ using the optical setup described previously $[20,22]$. A mirror galvanometer (GSV011, Thorlabs) was added to the setup to automatically control the low-pass filtered position $\left(f_{L P}=1 \mathrm{kHz}\right)$ of the laser spot on the position-sensitive detector (PSD) used to monitor cantilever vibration (measurement bandwidth $\sim 850 \mathrm{kHz}$ ). A Zurich Instruments HF2 lock-in amplifier was employed to record cantilever resonance spectra by sweeping a given range of excitation frequencies and demodulating the corresponding phase and amplitude (lock-in bandwidth $=4.38 \mathrm{~Hz}$, filter order $=24 \mathrm{~dB}$ /octave, 1000 data points). The setup was controlled using LabVIEW (National Instruments) and measurements were automated using the openBEB macro language [23]. The automation involved acquisition of spectra, adjusting the cantilever- 


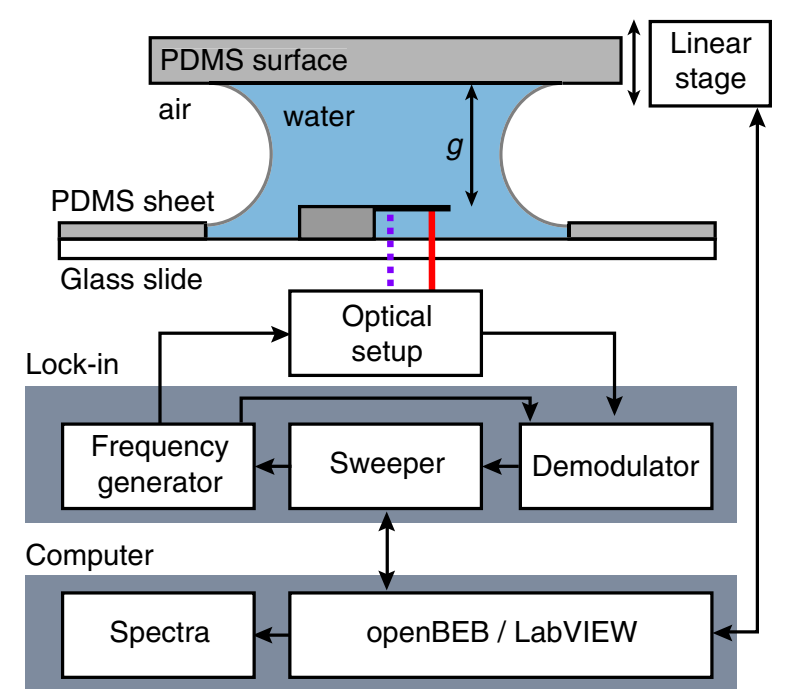

Figure 8 Experimental setup. Diagram of the experimental setup used to measure the dynamic response of a microcantilever vibrating in close proximity to a PDMS surface. The cantilever vibration was driven (purple dashed line) and detected (red solid line) optically using two laser beams. Cantilever chips were fixed on the bottom of a cavity used to confine the water. A PDMS surface attached to a motorized linear stage was moved down towards the cantilever, while continuously acquiring resonance spectra using a lock-in amplifier. The setup was controlled by software written in openBEB and LabVIEW.

surface gap, and adjusting the laser power and position of the laser spot on the positionsensitive detector using a proportional-integral-derivative (PID) controller. The whole setup was temperature controlled to $293 \mathrm{~K}$ within $\pm 0.2 \mathrm{~K}$.

Tipless silicon microcantilevers (NSC12/tipless/noAl, MikroMasch) with nominal dimensions of $250 \mu \mathrm{m} \times 35 \mu \mathrm{m} \times 2 \mu \mathrm{m}$ and calculated spring constants of $0.76 \mathrm{~N} / \mathrm{m}$ were employed. The data reported in the Additional file 1 was obtained using longer microcantilevers $(300 \mu \mathrm{m} \times 35 \mu \mathrm{m} \times 2 \mu \mathrm{m}$ and $350 \mu \mathrm{m} \times 35 \mu \mathrm{m} \times 2 \mu \mathrm{m})$ following the same protocol. A comparison of the different cantilevers is provided in the Additional file 1: Table S1. To improve reflectivity and avoid unspecific adsorption, $20 \mathrm{~nm}$ gold was coated at the bottom side of the cantilevers and they were passivated with short polyethylene glycol chains, as described previously [22].

The cavity containing the water was formed using PDMS (SYLGARD 184, Dow Corning) and a glass microscope slide (AA00000112E, Menzel-Gläser), exploiting surface tension forces (see Figure 8). The base was fabricated by reversibly bonding a $150 \mu \mathrm{m}$-thick PDMS sheet with a $10 \mathrm{~mm}$ wide circular hole at its center to the glass slide. The $300 \mu \mathrm{m}$-thick cantilever chip was attached to the glass slide at the center of the hole using UV curable glue (F-UVE-61, Newport). The thickness of the chip was sufficient $(H=8.6)$ to exclude any influence of the glass surface on cantilever dynamics. Furthermore, as the thickness of the PDMS sheet $(150 \mu \mathrm{m})$ was less than the thickness of the chip, access from above was retained. A flat upper cavity surface was fabricated by pouring degassed PDMS onto a silicon wafer to a thickness of about $5 \mathrm{~mm}$ and baking for 4 hours at $60^{\circ} \mathrm{C}$. The PDMS was subsequently removed from the wafer and cut to give a circular disk with a diameter of $15 \mathrm{~mm}$. The diameter exceeded all dimensions of the microcantilevers by at least an order of magnitude to avoid edge effects. The rougher surface of the disc was fixed to a kinematic mirror mount (KM05/M, Thorlabs), 
which was in turn mounted on an encoded piezo motor linear stage (CONEX-AG-LS2527P, Newport) with a nominal precision of $0.2 \mu \mathrm{m}$. The cavity allowed the cantilever to be immersed in $\sim 200 \mu \mathrm{L}$ of water.

The flat upper PDMS surface was manually aligned parallel to the cantilever. To do this, a piece of silicon wafer was attached to the surface by adhesion forces to render it reflective. The read-out laser was then focused on the silicon surface and detected by the PSD otherwise used to measure the cantilever deflection. The residual angular misalignment was estimated to be less than $1 \mathrm{mrad}\left(0.06^{\circ}\right)$. The same procedure was repeated after rotating the PSD by $90^{\circ}$ to align the angle perpendicular to the longitudinal axis of the cantilever.

To determine the coarse contact point, the surface was approached to the cantilever until a large deviation in the deflection signal was observed. Next, the surface was withdrawn to a distance where it had no influence on the cantilever vibration ( $g \approx$ $200 \mu \mathrm{m}, H \approx 6$ ). To adjust the gap, the motorized linear stage was operated in a closedloop configuration. After recording a spectrum the position was stored and the surface was moved closer to the cantilever. The step size was reduced as the gap decreased, to account for the non-linearity of squeeze-film damping. Next, for a more precise gap determination, the model of Tung et al. [14] was fitted to the frequency data of the fundamental mode (see Figure 4a) with parameters $f_{1, v a c}$ and a gap offset:

$$
\frac{f_{1}}{f_{1, \text { vac }}}=\left(1+\frac{\pi \rho_{f} b}{4 \rho_{c} h} \mathfrak{R}\left(\Gamma_{\text {Tung }}(R e, 2 H)\right)\right)^{-\frac{1}{2}}
$$

The offset was then subtracted from the z-position of the measurement to align the data. We emphasize that the definition of $H$ by Tung et al. [14] differs by a factor of two from Equation (1).

\section{Data analysis}

All data analysis was performed using custom scripts in IGOR Pro (Wavemetrics, see Additional file 1). Both amplitude and phase spectra contain the eigenfrequencies and quality factors of the vibrational modes. However, at small cantilever-surface gaps the resonance peaks in the amplitude spectrum become indistinguishable due to the strong peak broadening, i.e., low quality factors (see Figure 2). Furthermore, large differences in peak amplitude among higher modes of vibration complicate fitting and introduce dependencies on the initial parameters. In contrast, the phase shifts of each mode remain well resolved even at low quality factors. Thus, phase spectra were used to extract the modal eigenfrequencies and quality factors (see Figure 3). To weight each mode by the same amount on least squares fitting, the frequency spacing was transformed from linear $(p=1)$, i.e., equally spaced, to a power law according to

$$
f^{*}(m)=\left(\frac{m}{M-1}\left(f_{m=M}^{p}-f_{m=0}^{p}\right)+f_{m=0}^{p}\right)^{\frac{1}{p}}
$$

where $m$ is a data point in the spectrum ranging from 0 to $M-1, M$ the total number of points, $f_{m=O}$ the lowest and $f_{m=M}$ the highest frequency in the measured data and $p$ the power of the transformation required for each mode of vibration to be assigned an equal number of data points. The value of $p$ was estimated to be 0.514 from the calculated widths of the resonance peaks of all employed cantilevers in an unbounded fluid 
[21]. The phase values corresponding to the transformed frequencies $f^{*}$ were linearly interpolated from the measured data.

The following expression was used to extract the modal eigenfrequencies, $f_{n}$, and quality factors, $Q_{n}$, from the phase spectrum using a Levenberg-Marquardt algorithm (see Figure 3):

$$
\begin{aligned}
\phi(f) & =\phi_{c}\left(f, f_{1}, Q_{1}, \ldots, f_{N}, Q_{N}\right)+\phi_{t h}\left(f, \tau_{t h}\right)+\phi_{e l}\left(f, f_{e l}, c_{o f f}\right) \\
& =\sum_{n=1}^{N} \arctan \left(Q_{n} \frac{f_{n}^{2}-f^{2}}{f_{n} f}\right)-2 \pi f \tau_{t h}+\arctan \left(\frac{f_{e l}}{f}\right)+c_{o f f}
\end{aligned}
$$

where the cantilever response $\phi_{c}$ is the sum of damped harmonic oscillators with $f_{n}$ and $Q_{n}$ over all recorded modes $N, \phi_{t h}$ is the linear thermal lag due to photothermal excitation with time constant $\tau_{t h}$ [24] and $\phi_{e l}$ (center frequency $f_{e l}$ and offset $c_{o f f}$ ) is an empirical first-order filter that considers the phase responses of the measurement electronics. The filter center frequency $f_{e l}$ and the time constant $\tau_{t h}$ were determined on the first spectrum recorded far from the surface $(H » 1)$ and then held constant.

The linearized equation of motion for a cantilever of length $L$, width $b$, thickness $h$ and mass density $\rho_{c}$ is [16]:

$$
E I \frac{\partial^{4} Z(x, t)}{\partial x^{4}}+\mu_{c}\left(1+a_{m}\right) \frac{\partial^{2} Z(x, t)}{\partial t^{2}}+c \frac{\partial Z(x, t)}{\partial t}=F_{\text {drive }}(x, t)
$$

where $Z(x, t)$ is the z-direction flexural displacement at position $x$ along the cantilever beam at time point $t, E$ and $I=b h^{3} / 12$ the Young's modulus and area moment of inertia of the cantilever, $\mu_{c}=\rho_{c} b h$ the mass per unit length of the cantilever, $a_{m}$ the added mass coefficient quantifying the co-moving fluid mass relative to the cantilever mass, $c$ the sum of structural and viscous damping per unit length, $F_{\text {drive }}$ an external driving

Table 2 Parameters for the employed silicon cantilevers immersed in water

\begin{tabular}{lll}
\hline Cantilever properties & & \\
\hline$L$ & Length & $250 \mu \mathrm{m}$ \\
$b$ & Width & $35 \mu \mathrm{m}$ \\
$h$ & Thickness & $2 \mu \mathrm{m}$ \\
$\rho_{c}$ & Mass density & $2330 \mathrm{~kg} \cdot \mathrm{m}^{-3}$ \\
$\mu_{c}$ & Mass per unit length & $0.163 \mathrm{mg} \cdot \mathrm{m}^{-1}$ \\
$E$ & Young's modulus & $169 \mathrm{GPa}$ \\
$l$ & Area moment of inertia & $23.3 \mu \mathrm{m}^{4}$ \\
$Q_{n}$ & Quality factor of mode $n$ & \\
$f_{n}$ & Eigenfrequency of mode $n$ & $\mathrm{~Hz}$ \\
$f_{n, v a c}$ & Vacuum frequency of mode $n$ & $\mathrm{~Hz}$ \\
$a_{m}$ & Added mass coefficient & $\mathrm{Pa} \cdot \mathrm{s}$ \\
$c$ & Damping per unit length & \\
\hline Fluid properties & & $998.25 \mathrm{~kg} \cdot \mathrm{m}^{-3}$ \\
\hline$\rho_{f}$ & Mass density & $1.005 \mathrm{mPa} \cdot \mathrm{s}$ \\
\hline$\eta_{f}$ & Viscosity & \\
\hline Gap properties & & $\mathrm{m}$ \\
$H=g / b$ & Gap & \\
\hline
\end{tabular}


force per unit length. The parameters used for the following calculations are provided in Table 2. The added mass coefficients $a_{m}$ were calculated from the measured eigenfrequencies $f_{n}[2]$ :

$$
a_{m}=\left(\frac{f_{n, v a c}}{f_{n}}\right)^{2}-1
$$

The vacuum frequencies $f_{w v a c}$ for each mode $n$ were determined far from the surface $(H » 1)$, where the added mass coefficient can be calculated for higher modes, with normalized mode number $\kappa$, according to the theory by Van Eysden and Sader [21]:

$$
a_{m}=\frac{\pi \rho_{f} b}{4 \rho_{c} h} \mathfrak{R}\left(\Gamma_{\text {VanEysden }}(\operatorname{Re}, \kappa)\right) \text { for } H \gg 1
$$

The damping coefficients per unit length were calculated as $[7,16]$

$$
c=\mu_{c}\left(1+a_{m}\right) \frac{2 \pi f_{n}}{Q_{n}}
$$

\section{Additional file}

Additional file 1: Information on the data analysis routine and additional data on longer microcantilevers is provided (see Additional file 1).

\section{Abbreviations}

AFM: Atomic force microscopy; PDMS: Polydimethylsiloxane; PID: Proportional-integral-derivative (controller); PSD: Position-sensitive detector; SD: Standard deviation.

\section{Competing interests}

The authors declare that they have no competing interests.

\section{Authors' contributions}

$B A B$ and $T B$ conceived and designed the study. RK and BAB carried out the experiments. RK, TB and BAB programmed the control software. BAB analyzed the data and drafted the manuscript. All authors revised the manuscript and approved the final version.

\section{Acknowledgements}

The authors gratefully acknowledge Henning Stahlberg (C-CINA, Biozentrum, University of Basel) for financial support and providing facilities, Shirley Müller (C-CINA, Biozentrum, University of Basel) for critically reading and discussing the manuscript, Francois Huber and Hans Peter Lang (SNI, Institute of Physics, University of Basel) for their support on cantilever preparation, Stefan Arnold and Andrej Bieri (C-CINA, Biozentrum, University of Basel) for fruitful discussions. This work was supported by ARGOVIA grant NoViDeMo and Swiss National Science Foundation grant SNF 200020_146619.

Received: 25 July 2014 Accepted: 13 November 2014

Published online: 12 December 2014

References

1. Joshi S, Hung S, Vengallatore S: Design strategies for controlling damping in micromechanical and nanomechanical resonators. EPJ Tech Instrum 2014, 1:5

2. Basak S, Raman A, Garimella SV: Hydrodynamic loading of microcantilevers vibrating in viscous fluids. J Appl Phys 2006, 99:114906.

3. Harrison C, Tavernier E, Vancauwenberghe O, Donzier E, Hsu K, Goodwin ARH, Marty F, Mercier B: On the response of a resonating plate in a liquid near a solid wall. Sens Actuators A Phys 2007, 134:414-426.

4. Decuzzi P, Granaldi A, Pascazio G: Dynamic response of microcantilever-based sensors in a fluidic chamber. J Appl Phys 2007, 101:024303.

5. Garcia R, Herruzo ET: The emergence of multifrequency force microscopy. Nat Nanotechno/ 2012, 7:217-226.

6. Kawakami M, Taniguchi Y, Hiratsuka Y, Shimoike M, Smith DA: Reduction of the damping on an AFM cantilever in fluid by the use of micropillars. Langmuir 2010, 26:1002-1007.

7. Maali A, Cohen-Bouhacina T, Jai C, Hurth C, Boisgard R, Aimé J-P, Mariolle D, Bertin F: Reduction of the cantilever hydrodynamic damping near a surface by ion-beam milling. J Appl Phys 2006, 99:024908.

8. Ghatkesar MK, Barwich V, Braun T, Ramseyer J-P, Gerber C, Hegner M, Lang HP, Drechsler U, Despont M: Higher modes of vibration increase mass sensitivity in nanomechanical microcantilevers. Nanotechnology 2007, 18:445502. 
9. Spletzer M, Raman A, Reifenberger R: Elastometric sensing using higher flexural eigenmodes of microcantilevers. Appl Phys Lett 2007, 91:184103.

10. Dohn S, Sandberg R, Svendsen W, Boisen A: Enhanced functionality of cantilever based mass sensors using higher modes. Appl Phys Lett 2005, 86:233501.

11. Bao M, Yang H: Squeeze film air damping in MEMS. Sens Actuators A Phys 2007, 136:3-27.

12. Dareing DW, Yi D, Thundat $T$ : Vibration response of microcantilevers bounded by a confined fluid. Ultramicroscopy 2007, 107:1105-1110.

13. Green CP, Sader JE: Frequency response of cantilever beams immersed in viscous fluids near a solid surface with applications to the atomic force microscope. J Appl Phys 2005, 98:114913.

14. Tung RC, Jana A, Raman A: Hydrodynamic loading of microcantilevers oscillating near rigid walls. J Appl Phys 2008, 104:114905.

15. Grimaldi E, Porfiri M, Soria L: Finite amplitude vibrations of a sharp-edged beam immersed in a viscous fluid near a solid surface. J Appl Phys 2012, 112:104907.

16. Naik T, Longmire EK, Mantell SC: Dynamic response of a cantilever in liquid near a solid wall. Sens Actuators A Phys 2003, 102:240-254.

17. Rankl C, Pastushenko V, Kienberger F, Stroh CM, Hinterdorfer P: Hydrodynamic damping of a magnetically oscillated cantilever close to a surface. Ultramicroscopy 2004, 100:301-308.

18. Kim S, Kihm KD: Temperature dependence of the near-wall oscillation of microcantilevers submerged in liquid environment. Appl Phys Lett 2007, 90:081908.

19. Fornari A, Sullivan M, Chen H, Harrison C, Hsu K, Marty F, Mercier B: Experimental observation of inertia-dominated squeeze film damping in liquid. J Fluids Eng 2010, 132:121201.

20. Bircher BA, Duempelmann L, Lang HP, Gerber C, Braun T: Photothermal excitation of microcantilevers in liquid: effect of the excitation laser position on temperature and vibrational amplitude. Micro Nano Lett 2013, 8:770-774.

21. Van Eysden CA, Sader JE: Frequency response of cantilever beams immersed in viscous fluids with applications to the atomic force microscope: arbitrary mode order. J Appl Phys 2007, 101:044908.

22. Bircher BA, Duempelmann L, Renggli K, Lang HP, Gerber C, Bruns N, Braun T: Real-time viscosity and mass density sensors requiring microliter sample volume based on nanomechanical resonators. Anal Chem 2013, 85:8676-8683

23. Ramakrishnan C, Bieri A, Sauter N, Roizard S, Ringler P, Müller SA, Goldie KN, Enimanev K, Stahlberg H, Rinn B, Braun T: openBEB: open biological experiment browser for correlative measurements. BMC Bioinformatics 2014 $15: 84$.

24. Pini V, Tiribilli B, Gambi CMC, Vassalli M: Dynamical characterization of vibrating AFM cantilevers forced by photothermal excitation. Phys Rev B 2010, 81:054302.

\section{Submit your manuscript to a SpringerOpen ${ }^{\circ}$ journal and benefit from:}

- Convenient online submission

- Rigorous peer review

- Immediate publication on acceptance

- Open access: articles freely available online

- High visibility within the field

- Retaining the copyright to your article 